\title{
COMPARATIVE STUDY OF MODIFIED TENSION BAND WIRING VERSUS TENSION BAND THROUGH PARALLEL CANNULATED CANCELLOUS SCREWS IN PATELLA FRACTURES
}

\author{
Shrestha $P^{1^{*}}$, Chalise $P K^{1}$, Paudel $S R^{2}$
}

\section{Affiliation}

1. Department of Orthopedics and Trauma, Nepal Medical College Teaching Hospital, Kathmandu, Nepal

2. Department of Orthopaedics, Scheer Memorial Adventist Hospital, Banepa, Nepal

\section{ARTICLE INFO}

Received : 28 Februrary, 2019

Accepted : 27 November, 2019

Published : 31 December, 2019

(c) Authors retain copyright and grant the journal right of first publication with the work simultaneously licensed under Creative Commons Attribution License CC - BY 4.0 that allows others to share the work with an acknowledgment of the work's authorship and initial publication in this journal.

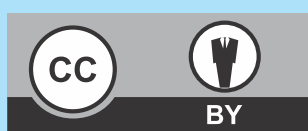

ORA 129

DOI: http://dx.doi.org/10.3126/bjhs.v4i3.27012

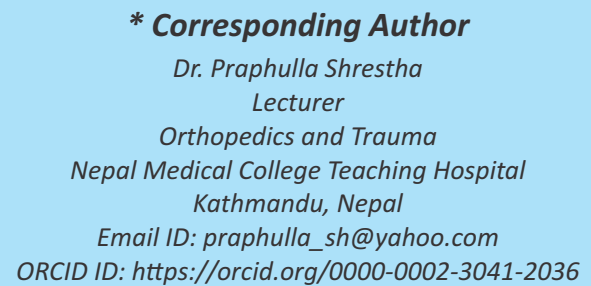

\section{Citation}

Shrestha P, Chalise PK, Paudel SR. Comparative Study of Modified Tension Band Wiring Versus Tension Band Through Parallel Cannulated Cancellous Screws in Patella Fractures. BJHS 2019;4(3)10: 777-781.

\section{ABSTRACT}

\section{Introduction}

Open reduction and internal fixation is indicated in patients with displaced patella fractures and loss of the extensor apparatus. Transverse fractures of the patella are commonly treated with modified tension band wire (MTBW). Loosening of the wires along with the implant construct and soft tissue irritation by the wires had already been reported. The newer technique, tension band wiring through cannulated cancellous screws (TBWCCS) is soft tissue friendly and gives better stability.

\section{Objective}

The objective of this study is to compare the outcome of treatment of patella fractures by modified tension band wiring with $\mathrm{k}$ wires and tension band wiring through parallel cannulated cancellous screws in terms of union rate, functional outcome and complication rate.

\section{Methodology}

We performed a comparative study between the two procedures from September 2017 to January 2019 at Nepal Medical College Teaching Hospital. Total of 40 patients, 20 in each group were enrolled in the study. All the patients were followed at regular intervals. Time taken for radiographic union was recorded. Variables of Modified Hospital for Special Surgery Knee Score (MHSSKS) were recorded and graded till the last follow-up at 24 weeks. Complications of each procedure were recorded. The data was analyzed using SPSS version 16.

\section{Result}

All fractures united at $12.20 \pm 3.03$ weeks in MTBW group and $11.20 \pm 2.78$ weeks in TBWCCS group. Complication rate was significantly higher in MTBW group. In TBWCCS group, 90\% patients had good to excellent MHSSKS score while in MTBW group, 75\% had good to excellent results at 24 weeks. Pain during walking was significantly better in TBWCCS group.

\section{Conclusion}

On the basis of our study, tension band wiring through cannulated cancellous screws is an effective method of treatment of patellar fractures.

\section{KEYWORDS}

Cannulated Cancellous Screws, Internal fixation, Patella fracture. 


\section{INTRODUCTION}

Surgery is indicated in patients of patella fractures with loss of extensor mechanism of the knee. ${ }^{1}$ Articular step $>2 \mathrm{~mm}$, fracture separation $>3 \mathrm{~mm}$, osteochondral fractures and fractures with loose bodies in the knee are among other indications for surgery. ${ }^{1}$ The surgical options in patella fractures are cerclage wiring, tension band wiring, cancellous screws, cannulated cancellous screws with anterior tension band, percutaneous cancellous screws, fiber wire fixation and cable pin fixation..$^{2-6}$ The recommended treatment of displaced transverse patella fracture is modified tension band wiring(MTBW). ${ }^{2}$ But complications like soft tissue irritation by wires, loosening, breakage and migration of the hardware leading to loss of reduction and fixation failure are reported. ${ }^{6}$ Some studies have shown that tension band through parallel cannulated cancellous screws(TBWCCS)is superior to MTBW. ${ }^{7,8}$ Screwsprovide compression at the fracture, less soft tissue irritation and it provides stability even in osteopenic bones. ${ }^{9}$

We compared the results of MTBW with TBWCCS in patella fractures in terms of time taken for union, function (Modified Hospital for Special Surgery Knee Score ${ }^{10}$ - pain, function and range of motion) and complication rates.

\section{METHODOLOGY}

The study was carried out in department of Orthopedics and Trauma of Nepal Medical College Teaching Hospital in Kathmandu, Nepal. It was a comparative study which was performed from September 2017 to January 2019. Total of 40 patients, 20 in each group were enrolled in the study. (Explanation: Expecting two weeks difference of recovery time with a standard deviation of 2.2 weeks at 0.05 level of significance and $80 \%$ power of test the sample size per group is 20).

All the patients who met the inclusion criteria were enrolled in the study. Adults of age more than 18 of any sexes with a recent $(<2$ weeks) isolated transverse patella fracture or minimally comminuted fracture that could be treated with either technique in the study was included in the study. Fractures displaced more than $3 \mathrm{~mm}, \geq 2 \mathrm{~mm}$ of articular incongruity and loss of extensor mechanism were included in the study. Patients with associated fractures around the knee or associated other injuries of the same or contra lateral leg and patients with pre-existing condition of the knee e.g. arthritis, advanced osteoarthritis, infective arthritis were excluded from the study. Patients unfit for surgery on the basis of pre-operative evaluation and checkup were also excluded from the study. Demographic data and history were taken relevant to the mode of injury and time since injury. All the patients were examined, and diagnosis was confirmed with radiographic examination. Baseline investigations were done and fitness for anesthesia and surgery was obtained preoperatively. Informed written consent was obtained from all patients preoperatively for surgery including for research inclusion also. Cases with odd serial numbers were treated with TBWCCS and cases with even serial numbers were treated with MTBW. All the patients were operated on next regular list. Standard antero-posterior and lateral radiographs was performed postoperatively. The knee range of motion exercises was started after few days post operatively as pain allowed. The patients were allowed to ambulate while bearing weight as tolerated on the initial post-operative period within few days after surgery. The patients were discharged after they were ambulatory. Isometric quadriceps exercises were encouraged as early as possible. Sutures were removed at 2 weeks postoperatively. Post-operative protocol was similar in both groups.

The patients were followed in outpatient's department at 2 , $6,12,18$ and 24 weeks. Time taken for radiographic union was recorded. Variables of Modified Hospital for Special Surgery Knee Score (MHSSKS)15 were recorded on every follow up. Knee score was calculated and graded. Failure of fixation was reported as a discredit to the procedure. The data was entered into the proforma and analyzed using SPSS version 16 . The t-test was used to compare average of the groups for time and various scores.

\section{RESULT}

The total number of patients in this study was forty with age ranging from 18 to 65 years. Out of these patients, 35 patients were male, and 5 patients were female, with male to female ratio of 9:1 in MTBW group 5.66:1 in TBWCCS group. The minimum age of patient was 18 years and maximum was 65 years in both the groups with a mean age of 38.97 years. The mean age of MTBW group was 41.15 years and in TBWCCS group was 36.8 years. Majority of the patients $(80 \%)$ sustained patella fracture after a road traffic accident. The motorcycle accidents were the commonest mode of injury in both groups. Few patients had car accidents which were also included in the road traffic accident category and $4(10 \%)$ patients had history of slip and fall and some had fall from stairs. Left side was the commonly involved side on both groups. Total 11 and 12 patients had patella fracture of the left side in MTBW group and TBWCCS group respectively. Patients were evaluated with radiographs for fracture healing during follow-up visits. The average healing time in MTBW group was $12.20 \pm 3.03$ weeks and in TBWCCS group was $11.37 \pm 2.78$ weeks with $p$ value of 0.28 i.e. the union time between two groups was similar and there was no difference statistically (Table 1 ). The MHSSKS of patients were calculated during follow-up visits and the final score at 24 weeks was calculated for all patients. Score was graded as excellent, good, fair and poor if the score was 90-85, 84-74, 73-65 and $<65$ respectively. In MTBW group, 15 patients had good to excellent grades and in TBWCCS group, 18 patients had good to excellent results. One patient with excellent outcome with union at twelve weeks is presented as an example in figure 1.Five patients had fair to poor results in MTBW group and in TBWCCS group only two patients had fair results and no poor results. Scores were significantly better in TBWCCS group as indicated by $p$ value $<0.05$ (Table 1). In Modified Hospital for Special Surgery Knee Score (MHSSKS) pain is divided as pain at rest and pain during walking. Both have 
been graded as none, mild, moderate and severe and 0, 5, 10 and 15 points has been given respectively. Range of motion at the knee has been given 1 point for every 8 degrees of movement in the scoring system. Pain during walking was present in 15 cases in MTBW groupand 5 patients in TBWCCS group and $p$ value was significant $(<0.05)$ (Table 1). Pain at rest was present in 6 patients in MTBW group and in 2 patients in TBWCCS groupand $p$ value was significant $(<0.05)$. Range of motion at knee was full in $12(60 \%)$ patients in both groups. Out of the remaining $8(40 \%)$ patients; in MTBW group, 5 patients had ROM $>120$ and 3 had $<120$ ond in TBWCCS group and all 8 patients had ROM $>120$ o (Table 1).There were no complications in our patients regarding anesthesia. Only three patients developed superficial wound infection, two from MTBW group and 1 from the TBWCCS group. Four patients from MTBW group developed painful hardware and one developed loosening and breakage of hardware (Figure 2) even though union was achieved in these patients and hardware were removed after union was achieved. There was no case of nonunion in this study.

\begin{tabular}{|c|c|c|c|c|}
\hline Variable & \multicolumn{2}{|c|}{ MTBW TBWCCS } & Combinec & d $p$ value \\
\hline \begin{tabular}{|l|} 
Variable \\
\end{tabular} & MTBW & \begin{tabular}{|l} 
TBWCCS \\
\end{tabular} & Combined & $p$ value \\
\hline \multirow{2}{*}{\begin{tabular}{|l} 
Sex - Male \\
- Female
\end{tabular}} & 18 & 17 & 35 & \\
\hline & 02 & 03 & 05 & \\
\hline \multirow[t]{2}{*}{ Side of involvement } & 09 & 08 & 17 & \\
\hline & 11 & 12 & 23 & \\
\hline \multirow[t]{3}{*}{\begin{tabular}{|l} 
Mode of injury \\
\end{tabular}} & 14 & 18 & 32 & \\
\hline & 03 & 01 & 04 & \\
\hline & 03 & 01 & 04 & \\
\hline - 08 weeks & 05 & 07 & 12 & 0.28 \\
\hline Union & 09 & 10 & 19 & \\
\hline-16 weeks & 06 & 03 & 09 & \\
\hline \multirow[t]{4}{*}{ MHSSKS (Knee score) } & 05 & 08 & 13 & 0.29 \\
\hline & 10 & 10 & 20 & \\
\hline & 02 & 02 & 04 & \\
\hline & 03 & 00 & 03 & \\
\hline \multirow[t]{4}{*}{ Pain during walking } & 01 & 00 & 01 & 0.01 \\
\hline & 03 & 01 & 04 & \\
\hline & 11 & 04 & 15 & \\
\hline & 05 & 15 & 20 & \\
\hline \multirow[t]{4}{*}{ Pain at rest } & 00 & 00 & 00 & 0.20 \\
\hline & 02 & 00 & 02 & \\
\hline & 04 & 02 & 06 & \\
\hline & 14 & 18 & 32 & \\
\hline & 01 & 00 & 01 & 0.43 \\
\hline $\begin{array}{r}\text { Range of Motion (ROM) } 80 \text { degrees } \\
96 \text { degrees }\end{array}$ & 01 & 00 & 01 & \\
\hline 112 degrees & 01 & 00 & 01 & \\
\hline 120 degrees & 00 & 02 & 02 & \\
\hline 128 degrees & 03 & 02 & 05 & \\
\hline 136 degrees & 02 & 04 & 06 & \\
\hline 144 degrees & 12 & 12 & 24 & \\
\hline \multirow{3}{*}{\begin{tabular}{|l} 
Complications - Superficial infection \\
- Painful hardware \\
- Loosening or hardware breakage
\end{tabular}} & 02 & 01 & & 0.01 \\
\hline & 04 & 00 & & \\
\hline & 01 & 00 & & \\
\hline
\end{tabular}

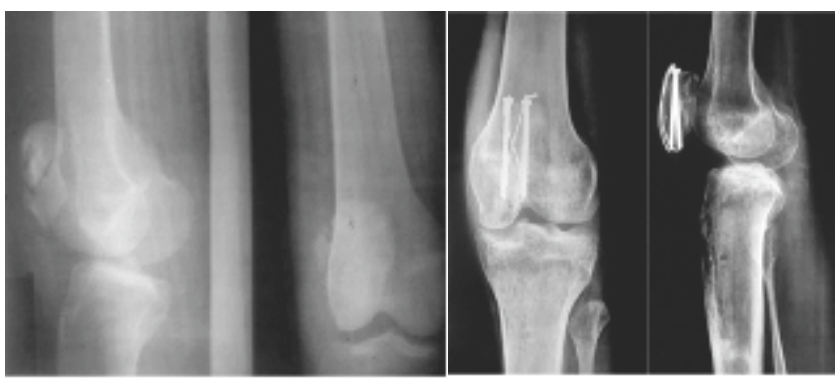

Figure 1: Fracture of patella with articular step treated with TBWCCS at 12 weeks with union

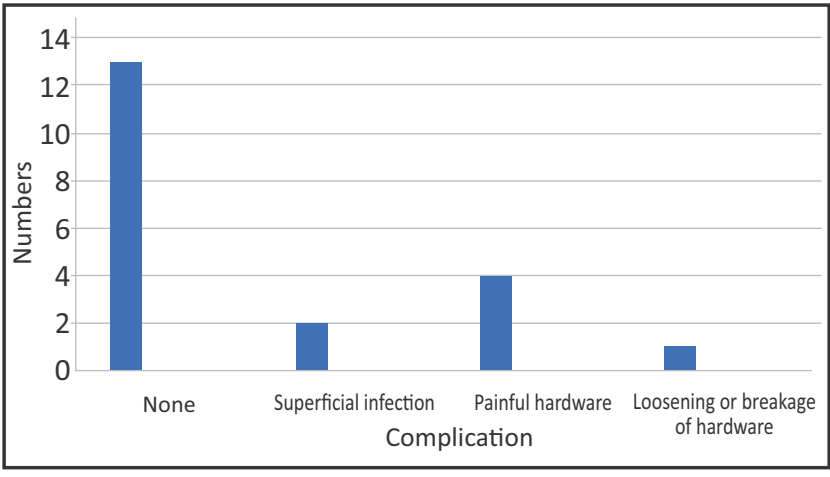

Figure 2: Complications of MTBW group

\section{DISCUSSION}

Traditionally, the common and recommended method of internal fixation of patella fractures is modified anterior tension band wiring. ${ }^{2}$ The distraction forces at the fracture site are converted into the compressive forces by tension band wire. ${ }^{2}$ Irritation of the local soft tissues by the impingement of the k-wire ends is a frequent complication reported in this type of fixation. ${ }^{10}$ Different surgeons have tried to modify this technique to reduce these complications. ${ }^{9,11}$ Some authors have reported both clinically and biomechanically, cannulated cancellous screws causes less soft tissue irritation, gives better stability and provides compression forces at the fracture site. By passing the stainless-steel wire through the cannulated screws and by figure of eight configuration anterior to the patella provides tension band principle in this type of fixation. ${ }^{7,9,12}$

In this study the maximum number of patients in both the groups were males, 18 (90\%) in MTBW group and 17 (82.35\%) in TBWCCS group. This can be explained by our cultural setup where males have a more active lifestyle and remain out-door more often to earn their living, while females stay mostly indoor. Road traffic accidents were the major cause of injury in both groups, $70 \%$ in MTBW group and $90 \%$ in TBWCCS group. Two wheelers like motorcycle and scooters are a very common mode of transportation in Nepal. Motorcycle riders are very prone to injuries and a major part of the skeletal injuries are due to motorcycle accidents in Nepal. ${ }^{13}$

Three of the patients ( 2 in MTBW group and 1 in TBWCCS group)i.e. $7.5 \%$ of patients developed postoperative infection. These superficial wound infections were treated with antibiotics and regular wound care. In different studies, the rate of infection after surgical treatment in patella fractures is $3-10 \% .{ }^{14-16}$ The infection rate in this study is similar to those studies and the infection was dealt with prolonged antibiotic treatment and care of the wound.

The average time of fracture union was $12.20 \pm 3.03$ \& $11.20 \pm 2.78$ weeks in MTBW and TBWCCS group respectively without significant statistical difference. In the study done by Berg, ${ }^{9}$ in which he has studied the outcome of tension band wire with cannulated cancellous screws only, the time to fracture union was 13 weeks on average which is similar to 
the time of union in this study. In the study by Khan I et al which studied only the outcome of TBW with cannulated cancellous screws, fracture union time is about 10.7 weeks in average. ${ }^{12}$ (Table 1 ) In the study by Tan et $a l,{ }^{15}$ which compared the MTBW and TBW with CCS, they had union time of about 10 weeks in average in both groups. The results of Modified Hospital for Special Surgery Knee score (MHSSKS) was good to excellent in $90 \%$ and $75 \%$ in TBWCCS group and MTBW group respectively. Fair to poor MHSSK Score was seen in 5 patients in MTBW group while 2 patients in TBWCCS group had fair results and no patient had poor results. Scores were better in TBWCCS group, but it is not statistically significant (Table 1). The pain during walking is better in TBWCCS group and was statistically significant (Table1). This can be attributed to the less soft tissue irritation by the screws in comparison to the $\mathrm{k}$ wires. As a result, patients in TBWCCS group had less pain and better function, and better quality of fixation allowing better range of motion at the knee. In the study by Catalano et al, the MHSSK Score is good to excellent in $73-90 \%$ of patients. ${ }^{16}$ The study of Bergon tension band through cannulated screw fixation had $70 \%$ good to excellent modified HSS knee score. ${ }^{12}$ This result was not comparable to our study which had $90 \%$ good to excellent outcome in patients treated with the same technique. Berg had included patients with other pre-existing conditions in the fractured limb which might be the reason of the difference of our study with his. Polio affected limbs and other diseases were also included in that study. We excluded the patients with other conditions in the fractured limb. Tan $\mathrm{H}$ et al showed good to excellent Bostman scores in $90 \%$ in MTBW group and $100 \%$ in TBWCCS group in their study. ${ }^{20}$ Good to excellent Bostman scores in $100 \%$ cases were shown by Khan l et al. ${ }^{12}$

Total of seven patients in MTBW group had complications including superficial infection, painful hardware and loosening and breakage of hardware while only one patient in TBWCS group had complication which was superficial infection. In this study, in MTBW group, five patients had hardware problems out of which $4(20 \%)$ had painful hardware and 1 (5\%) had loosening \& breakage of hardware. (Figure 3) The reported rate of painful hardware after patella fracture fixation is $15 \%$ which was similar in our study in MTBW group. ${ }^{18-19}$ In TBWCCS group, only 1 patient had superficial infection and no other complications were seen. There is statistically significance between the rate of complications than the TBWCCS group. MTBW group had higher rate of complications than TBWCCS group. Loosening of hardware and loss of reduction occurred in 0-20\% cases in previous studies. ${ }^{15,17}$ One patient (5\%) in MTBW group in our study had loosening of hardware at 16 weeks and breakage of hardware at 24 weeks follow-up although the fracture united in this case also.

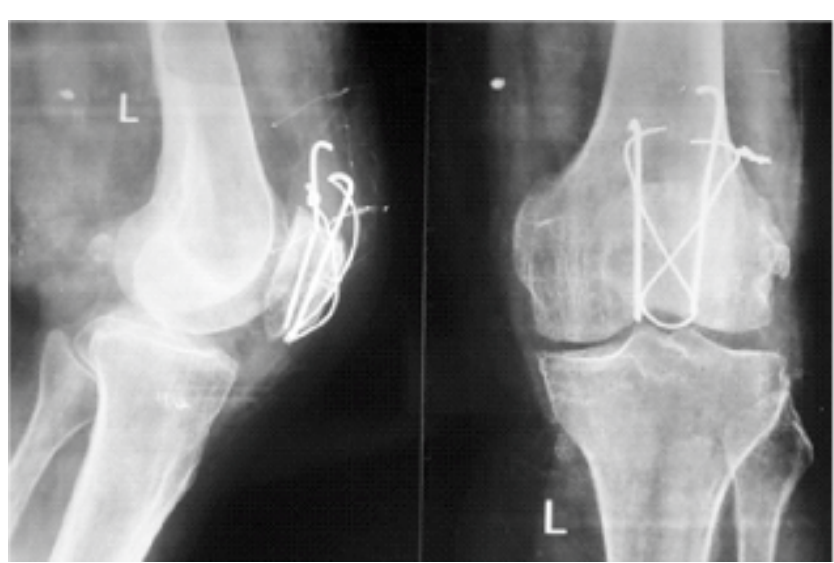

Figure 3: At 24 weeks, a patient had loosening and breakage of wires

In our initial few surgeries of TBWCCS, the screw size was always longer appearing in the postoperative radiographs. Subsequently we started using shorter screws than the actual measurement but keeping in mind that the screw threads always crossed the fracture site. It is always necessary for the screw threads to cross the fracture site so that the threads should lie only on the far fragment during insertion to achieve compression at the fracture site.

\section{CONCLUSION}

This study concludes that tension band wiring through cannulated cancellous screws (TBWCCS) is an effective method of treatment of patella fractures with comparable rate of union and better functional outcome along with decreased rate of complications when compared with modified tension band wiring (MTBW).

\section{RECOMMENDATIONS}

On the basis of this study, we recommend that tension band wiring through parallel cancellous cannulated screws in patella fractures can be used as a better alternative to modified tension band wiring whenever possible. The use of shorter screws than actual measurement is recommended to avoid placement of longer screws which may avoid soft tissue irritation.

\section{LIMITATIONS OF THIS STUDY}

The sample size was small so further studies with a larger sample size would be better to establish the conclusion.

\section{ACKNOWLEDGEMENT}

The authors would like to thank Dr Suman Thapa Magar for his help in data management.

\section{CONFLICT OF INTEREST}

The authors declare that they have no conflict of interest related to the publication of this manuscript.

\section{FINANCIALDISCLOSURE}

There are no financial conflicts to disclose. 


\section{REFERENCES}

1. Harris RM. Fractures of the Patella and injuries to the Extensor Mechanism. In: Bucholz RW, HarrisHeckman JD C-BC, ed. Rockwood and Green's Fractures in Adults. Sixth. Lippincott Williams and Wilkins; 2006:1970-1995.

2. Müller ME, Allgöwer $M$, Allgower $M$, Schneider R WH. Manual of Internal Fixation: Techniques Recommended by the AO-ASIF Group. Springer Science \& Business Media; 1991.

3. Khan I, Dar MY, Rashid S, Butt MF. Internal fixation of transverse patella fractures using cannulated cancellous screws with anterior tension band wiring. Malaysian Orthop J. 2016;10(2):21-26. DOI:10.5704/MOJ.1607.005.

4. Cho J-H. Percutaneous cannulated screws with tension band wiring technique in patella fractures. Knee Surg Relat Res. 2013;25(4):215219. DOI:10.5792/ksrr.2013.25.4.215.

5. Camarda L, La Gattuta A, Butera M, Siragusa F, D’Arienzo M. FiberWire tension band for patellar fractures. J Orthop Traumatol. 2016;17(1):75-80. DOI:10.1007/s10195-015-0359-6.

6. Mao N, Liu D, Ni H, Tang H, Zhang Q. Comparison of the cable pin system with conventional open surgery for transverse patella fractures knee. Clin Orthop Relat Res. 2013;471(7):2361-2366. DOI:10.1007/s11999-013-2932-8.

7. Burvant JG, Thomas KA, Alexander R, Harris MB. Evaluation of methods of internal fixation of transverse patella fractures: a biomechanical study. J Orthop Trauma. 1994;8(2):147-153. PMID: 8207572

8. Cekin T, Tukenmez M, Tezeren G. [Comparison of three fixation methods in transverse fractures of the patella in a calf model]. Acta Orthop Traumatol Turc. 2006;40(3):248-251. PMID: 16905899

9. Berg EE. Open reduction internal fixation of displaced transverse patella fractures with figure-eight wiring through parallel cannulated compression screws. J Orthop Trauma. 1997;11(8):573-576. PMID: 9415863
10. Insall JN, Dorr LD, Scott RD, Scott WN. Rationale of The Knee Society Clinical Rating System. 1989. PMID: 2805470

11. Wu CC, Tai $\mathrm{CL}$, Chen WJ. Patellar tension band wiring: a revised technique. Arch Orthop Trauma Surg. 2001;121(1-2):12-16. PMID: 11195111

12. Khan I, Dar MY, Rashid S, Butt MF. Internal fixation of transverse patella fractures using cannulated cancellous screws with anterior tension band wiring. Malaysian Orthop J. 2016;10(2):21-26. DOI:10.5704/MOJ.1607.005.

13. Huang L, Poudyal AK, Wang N, Maharjan RK, Adhikary KP, Onta SR. Burden of road traffi c accidents in Nepal by calculating disabilityadjusted life years. Fam Med Community Heal Orig Res 179 Fam Med Community Heal. 2017;5(3):179-187. DOI: https://doi.org/ 10.15212/FMCH.2017.0111

14. Böstman $O$, Kiviluoto $O$, Nirhamo J. Comminuted displaced fractures of the patella. Injury. 1981;13(3):196-202. DOI:10.1016/00201383(81)90238-2.

15. Smith ST, Cramer KE, Karges DE, Watson JT, Moed BR. Early complications in the operative treatment of patella fractures. J Orthop Trauma. 1997;11(3):183-187. PMID: 9181501

16. Hung LK, Chan KM, Chow YN, Leung PC. Fractured patella: operative treatment using the tension band principle. Injury. 1985;16(5):343347. DOI:10.1016/0020-1383(85)90144-5.

15. Tan H, Dai P, Yuan Y. Clinical results of treatment using a modified Kwire tension band versus a cannulated screw tension band in transverse patella fractures: A strobe-compliant retrospective observational study. Med (Baltimore). 2016;95(40):e4992. PMID: 27749556

16. Catalano JB, lannacone WM, Marczyk S, et al. Open fractures of the patella: long-term functional outcome. J Trauma. 1995;39(3):439444. PMID: 7473905

17. Levack B, Flannagan JP, Hobbs S. Results of surgical treatment of patellar fractures. J Bone Joint Surg Br. 1985;67(3):416-419. PMID: 3997951 